\title{
A Spasticity Assessment Method for Voluntary Movement using Data Fusion and Machine Learning
}

yan chen

south china university of technology https://orcid.org/0000-0001-7650-3307

\section{Song Yu}

south china of university of Technology

\section{Qing Cai}

The Third Affiliated Hospital of Sun Yat-sen University

\section{Shuangyuan Huang}

South China University of Technology

\section{$\mathrm{Ke} \mathrm{Ma}$}

South China University of Technology

Haiqing Zheng

The Third Affiliated Hospital of Sun Yat-sen University

Longhan Xie ( $\triangle$ melhxie@scut.edu.cn )

https://orcid.org/0000-0002-5137-1413

\section{Research}

Keywords: Spasticity, Data Fusion, Machine Learning, Classification

Posted Date: March 27th, 2020

DOI: https://doi.org/10.21203/rs.3.rs-19134/v1

License: (c) (i) This work is licensed under a Creative Commons Attribution 4.0 International License.

Read Full License

Version of Record: A version of this preprint was published on March 1st, 2021. See the published version at https://doi.org/10.1016/j.bspc.2020.102353. 


\title{
A Spasticity Assessment Method for Voluntary Movement using Data Fusion and Machine Learning
}

\author{
Yan Chen ${ }^{1}$, Song $\mathrm{Yu}^{1}$, Qing $\mathrm{Cai}^{3}$, Shuangyuan Huang${ }^{1}, \mathrm{Ke} \mathrm{Ma}^{2}$, Haiqing Zheng ${ }^{3}$ and \\ Longhan $\mathrm{Xie}^{\mathbf{1}^{*}}$ \\ ${ }^{1}$ Shien-Ming Wu School of Intelligent Engineering, South China University of Technology, Guangzhou, 511442, China \\ ${ }^{2}$ School of Mechanical and Automotive Engineering, South China University of Technology, Guangzhou, 510640, China \\ ${ }^{3}$ The Third Affiliated Hospital, Sun Yat-sen University, Guangzhou, 510630, China \\ Corresponding author: Longhan Xie (e-mail: melhxie@scut.edu.cn).
}

\begin{abstract}
Background: Spasticity is a common complication of stroke. Effective spasticity management can improve patients' recovery efficiency and reduce patients' pain. The present clinical spasticity rating scale exhibits subjectivity and a ceiling effect, which makes it difficult to evaluate spasm objectively and to clinically analyze the pathological mechanism of spasticity. The sensor-based quantitative evaluation method is an effective substitute for the clinical spasm rating scale, but currently, it mainly focuses on the spasm evaluation of passive motion. The study of spasmodic state under active exercise can provide a basis for treatment and rehabilitation training, but the evaluation method of spasmodic state under active exercise has not yet been established. Therefore, we combine inertial measurement unit (IMU) and surface electromyography (sEMG) to test the feasibility of assessing spasticity patterns in stroke patients during voluntary movement.
\end{abstract}

Methods: Nine stroke patients with varying degrees of spasticity and four healthy subjects performed isometric elbow exercises. sEMG and kinematics signals were recorded for all participants. The Empirical Mode Decomposition (EMD) algorithm and double threshold algorithms were used to separate sEMG of involuntary muscle activation from voluntary activation. Then, feature extraction and feature fusion were performed. Four common machine learning algorithms are used to monitor and evaluate spasticity patterns. The validity of the proposed method is verified by comparing the classification accuracy of four machine learning models.

Results: Cross-validation yielded high classification accuracies (F1-score $>0.88$ ) for all four machine learning classifiers in assessing spasticity patterns. The highest detection performance was obtained using the Random Forest algorithm (average accuracy $=0.979 ;$ macro-F1 $=0.976$ ).

Conclusions: We present a novel method for assessing post-stroke spasticity based on voluntary movement and machine learning. Good classification performance verifies the feasibility of evaluating spasticity patterns by our method. Reliable classification accuracy achieved by the machine learning algorithms indicated the potential to evaluate spasticity patterns using IMU and sEMG when stroke survivors perform voluntary movements.

Keywords: Spasticity, Data Fusion, Machine Learning, Classification 


\section{Background}

Spasticity is an abnormal muscle tone caused by injury of the pyramidal and parapyramidal tract, which is the most common complication of stroke. Spasticity describes involuntary muscle hyperactivity in the presence of central paresis [1],[2]. According to the Brunnstrom Approach[3], hemiplegic patients begin to experience spasticity in phase II; spasticity is the most severe in phase III and disappears in phase IV [4]. The development of poststroke spasticity is related to neuronal plastic changes within the central nervous system after the initial injury [5],[6]. Therefore, in the process of hemiplegia rehabilitation, the degree of spasticity is an important factor of the therapist's clinical intervention and rehabilitation training.

The Modified Ashworth Scale (MAS) is the most widely used convulsion scale in clinics [7]. However, studies have shown that the semi-quantitative descriptions of MAS lead to ambiguous results [8],[9],[10]. MAS give a fuzzy boundary of spasticity degree. The intervals between each degree may not equal. Those semi-quantitative descriptions cause the therapist made subjective judgment. At present, subjective problems existed in all of the spasticity clinical scales [11],[12], which make it difficult to evaluate spasticity objectively and quantitatively. Quantitative assessment of spasticity is helpful to the study of spastic pathology and plays a pivotal role in formulating rehabilitation plans or adjusting doses of antispasmodic drugs. However, due to the complexity and multifactorial nature of spasticity, how to quantify spasticity remains a challenge and unresolved problem [13].

To improve the objectivity and reliability of the assessment of spasticity, numerous methods have been developed. These different evaluation methods can be divided into passive motion evaluation methods and voluntary motion evaluation methods. The evaluation method based on passive motion is the mainstream quantitative evaluation method at present. By collecting the biological signals of patients, researchers extracted features and analyzed the correlation between the features and the traditional clinical scales, such as MAS. For example, the inertial sensor or the exoskeleton machine can be used to provide biomechanical information, such as the counter torque and the angle of joint motion in the process of passive stretching [14],[15],[16]. In addition, H-reflex and H/M ratio [17] are determined based on electromyography signals and using Shear Wave Elastography [18],[19] to directly measure muscle stiffness. Although the mechanical signalbased approach has good interpretability, the absence of muscle activation analysis makes it difficult to identify the disturbance caused by contractures. In current studies, it is generally believed that TSRT [20],[21],[22] based on inertial measurement unit (IMU) and surface electromyography (sEMG) fusion exhibits reliable clinical validity. [23] shows that the neural component is the domination factor of spasticity assessment for most patients in the passive stretch test. Combining IMU and sEMG can achieve information complementarity, thus improving the assessment accuracy of stroke patients [24]. Spasticity assessment of passive movement can be achieved by combining kinematic data and sEMG.

Although passive stretch is fundamental in clinical measurements of spasticity, it cannot assess the effect of spasticity on motion. Spasticity may be of some benefit in the maintenance of muscle tone [25], but intervention is necessary if spasms affect movement. It is difficult to observe the effect of spasticity on motor function. The study of spasticity under active movement is helpful to explore the pathological mechanism of spasticity [11]. Spasmodic assessment under active exercise can improve the efficiency of rehabilitation training. On one hand, therapists can develop more effective rehabilitation plans or therapeutic interventions for patients [26]. On the other hand, to make telemedicine possible, evaluation scenarios are not limited to hospitals. Moreover, the evaluation of spasm under active motion provides sensory feedback for the rehabilitation robot, which is conducive to improving the safety of the rehabilitation robot.

However, few studies focus on the spasticity assess based on patients' voluntary movements. The main reason for the lack of research is that the Lance's definition of spasticity [27] is based on passive movements. However, the Lance definition is narrow in pure motor disorders. It does not account for the measurement/description of spasticity under both active and passive conditions [2]. [28],[29] studied the relationship between active hypokinesis and abnormal coordination. It has been proved demonstrated that abnormal coordinated intervention can reduce the spasticity of active movement. [30] shows that voluntary contraction has a significantly correlates with passive stretch. Temporal features of movement can be potential targets for rehabilitation in individuals with upper limb spasticity after stroke [31]. Although the above studies have confirmed the feasibility of evaluating spasticity under active motion, none of these studies have established an evaluation model for spasm. To our knowledge, no studies have established the spasticity status recognition model of isotonic exercise.

Therefore, the purpose of this study was to investigate the feasibility of spasticity assessment 
based on isotonic exercise. The main contributions of this paper are as follows:

1. An algorithm to extract useful features of sEMG and kinematics data under active movement was proposed.

2. A spasmodic evaluation model under active motion was proposed with satisfactory accuracy (F1-score > 0.9).

3. A practical approach can be used to evaluate spasticity and refine the assessment of rehabilitation training, which can be applied to clinical assessment and potential applications in rehabilitation robotics and telemedicine.

Extensive experiments were conducted to validate the performance of the proposed methods. The level of spasticity was assessed by measuring the patient's elbow flexion and extension movements. A spasticity evaluation model of patients' isotonic contraction of elbow flexion and extension is investigated in this work. Specifically, the Empirical Mode Decomposition (EMD) algorithm was used to extract the characteristics of sEMG in the isotonic contraction process of stroke patients with spasticity, and a spasticity grading evaluation model was established based on random forest. Model performance was validated using isotonic exercise data of stroke patients.

\section{Methods}

Participants

Thirteen subjects (four health subjects and nine stroke patients with spasticity) were recruited to participate in the experiments. Patient information is shown in Table 1. Consent to participate (i.e., informed consent) was obtained from all the participants to follow the protocol approved by the Guangzhou First People's Hospital Department of Ethics Committee. All research was performed in accordance with the Declaration of Helsinki. The stroke participants met the following criteria. Inclusion criteria were as follows:

1) no therapeutic intervention was performed within 2 weeks;

2) stroke survivor either in the subacute (between 1 to 6 months post stroke) or chronic (over 6 months post stroke) stage of recovery;

3) a fair to good cognitive level (Mini Mental State Examination (MMSE) score $\geq 24$ );

4) Ability to perform the required motions.

Exclusion criteria were as follows:

1) upper limb pain $>4 / 10$ on a Visual Analogue Scale (VAS) [32];

2) upper limb spasticity $\geq 3$ on the MAS [33].

Data acquisition

Briefly, sEMG data were acquired using a commercial EMG acquisition system (Ultium ${ }^{\mathrm{TM}}$ Biomechanics
System, Noraxon Ltd, USA) with a frequency of 2000 Hz. With an amplitude range of 100-5000 uV and a frequency component of $0-500 \mathrm{~Hz}$ [35], sEMG data were amplified 1000 times. sEMG data collection was performed strictly in accordance with the recommended standards [34],[35]. sEMG data from three superficial muscles that are responsible for the main movements in the isotonic exercise task used in this article were recorded. As shown in figure 1(a), these muscles included the brachioradialis (BR), biceps brachialis (BB), triceps brachialis (TB). The surface electrode material was $\mathrm{AgCl}$, and the distance between the electrodes was $2 \mathrm{~cm}$. The direction of the electrodes was parallel to the muscle fibers. Prior to placing the surface electrodes, we wiped the skin surface with alcohol to reduce skin impedance. Then, the 3 sensorunits of the sEMG acquisition instrument were used to collect original sEMG of BM, BB and TB. Moreover, the sensor-unit of EMG acquisition system is capable of collecting not only sEMG but also IMU data. Therefore, the 3 sensor-units simultaneously collected IMU data and sEMG at a frequency of $200 \mathrm{~Hz}$.

Table 1 Demographic and clinical characteristics $(\mathrm{N}=9)$

\begin{tabular}{ccccccc}
\hline & $\begin{array}{c}\text { Ag } \\
\text { e }\end{array}$ & Sex & $\begin{array}{c}\text { Affected } \\
\text { Side }\end{array}$ & $\begin{array}{c}\text { Brunn } \\
\text {-strom }\end{array}$ & $\begin{array}{c}\text { Month } \\
\text { post } \\
\text { stroke }\end{array}$ & $\begin{array}{c}\text { Spasti- } \\
\text { city } \\
\text { degree }\end{array}$ \\
\hline P1 & 36 & M & Right & II & 9 & $1+$ \\
P2 & 66 & M & Left & III & 6 & $1+$ \\
P3 & 40 & M & Right & III & 4 & 2 \\
P4 & 31 & M & Right & IV & 4 & 2 \\
P5 & 55 & F & Left & II & 4 & 2 \\
P6 & 52 & M & Right & II & 4 & $1+$ \\
P7 & 58 & M & Right & III & 8 & 1 \\
P8 & 65 & M & Right & III & 9 & $1+$ \\
P9 & 64 & M & Left & I & 2 & 1 \\
\hline
\end{tabular}

Experimental protocols

As shown in Figure 1(b), a guide-assisted motion device consisting of a guide-track and a slider was used to assist patients to achieve the maximum range of motion. To reduce the influence of gravity on the flexion and extension range of the elbow, subjects flex and extend their elbows around a vertical axis on a horizontal plane. Subjects naturally sat at the table and adjusted the height of the table such that their forearms were parallel to the table. The slide track is placed on the table and adjusted such that the subject can naturally and comfortably hold the handle of the slide block and maintain maximum elbow flexion. Subjects hold or clamped the handle (patients with poor grasp) of the slider. During the isotonic exercise task, as shown in Figure 1(a), subjects first stay relaxed at the position of maximum elbow flexion for 2-3 s. Then, subjects 
moved from the position of maximum elbow flexion to the position of maximum elbow flexion. Thereafter, subjects relaxed to the position of maximum elbow flexion for 2-3 s. Finally, subjects moved back to the position of maximum elbow flexion. To avoid extra shoulder abduction, the tester stretches the end of the motion to the elbow, i.e., the range of motion is approximately $80^{\circ}-100^{\circ}$ [16]. Prior to performance of the isotonic exercise task, subjects performed 3-5 practice trials to ensure that they understood the task. Each subject performed the isotonic exercise task with his/her affected side. Each task was repeated 15 times at a self-selected speed. To avoid fatigue, the subjects were allowed 30-60 s of rest between two isotonic exercise tasks.

\section{Data processing}

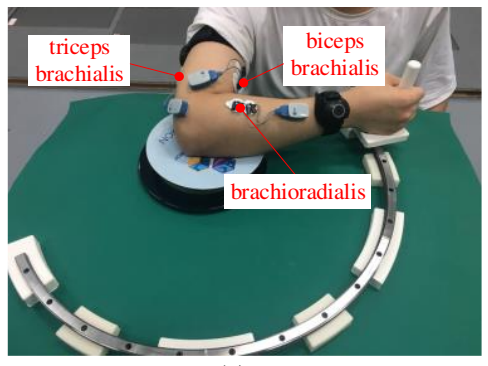

(a)
Data processing was implemented with MATLAB 2018b (The MathWorks Inc., Natick, USA). Figure 2 shows the flow chart of data acquisition, preprocess and model training in this paper. The initial 1-second data of all task sessions were removed to prevent the noise caused because the subject was not relaxed. Then, the original data of upper limb sEMG and IMU data were processed separately. Using a hamming window, the data dimension of sEMG signal and IMU data were processed to obtain the feature values at time $t$ window. The sEMG feature values of every muscle constituted the feature eigenvector $\boldsymbol{X}_{\boldsymbol{t}}$. IMU data were used to calculate the motion angles of elbow joint, and the kinematics feature of elbow joint constituted the vector $\boldsymbol{L}_{\boldsymbol{t}}$. Each training vector is calculated as: $\boldsymbol{T}_{\boldsymbol{t}}=$ $\left\{X_{t}, L_{t}\right\}$.

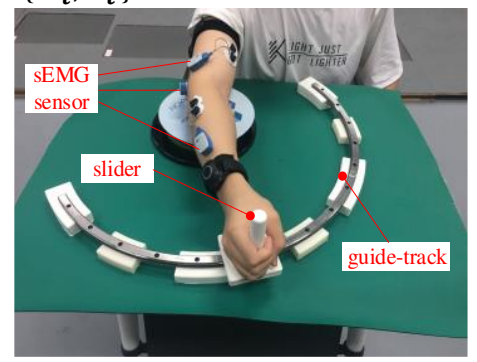

(b)

Fig. 1 Flowchart of the proposed methodology. (a) The position of the surface electrodes placed on the upper limb. (b) The experimental setup.

Data Preprocessing

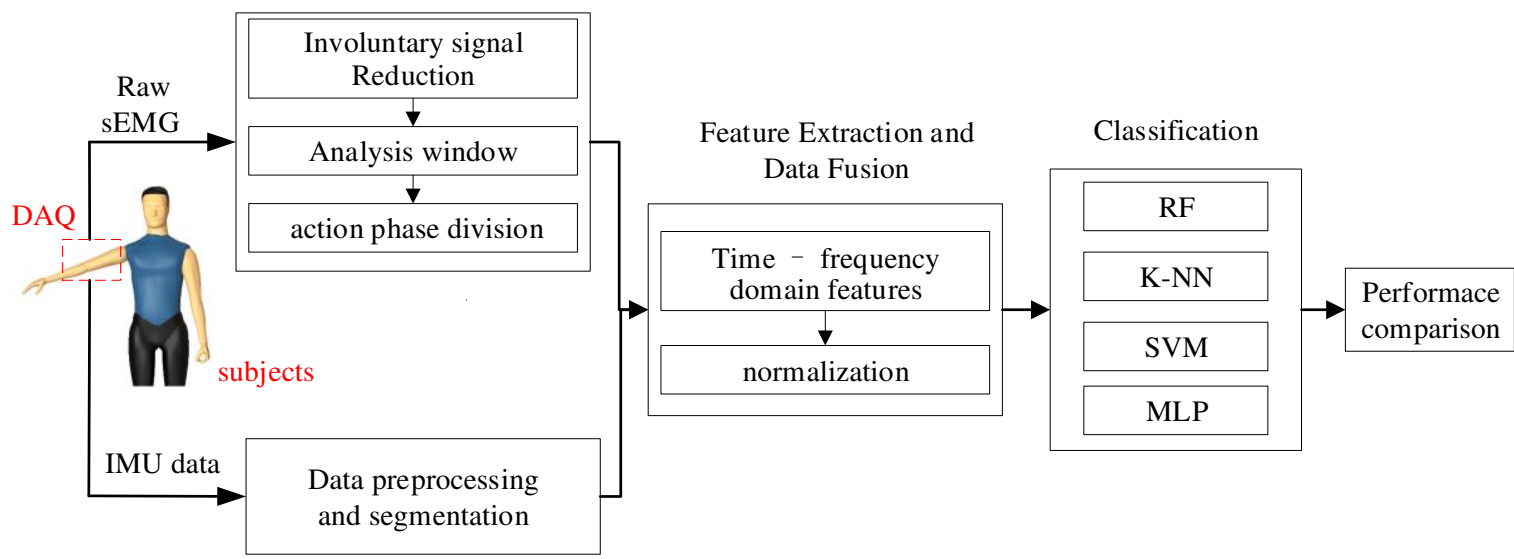

Fig. 2 Flowchart of the proposed methodology.

As shown in Figure 2, sEMG processing consisted of three parts: involuntary signal reduction, action phase division and feature extraction.

\section{Involuntary signal reduction}

During task execution, the sEMG included not only the voluntary active part of the patient but also the involuntary motor activity caused by the spasticity. Involuntary muscle movement increases the signal-tonoise ratio of the signal, which makes it difficult to distinguish the start and end time of voluntary movement. Thus, involuntary motor activity should be removed because it makes it more difficult to analyze the state of muscle activation under active operation in combination with kinematics characteristics. During the involuntary signal separation, EMD was performed to remove the baseline noise. Details of Empirical Mode Decomposition are presented in [36]. In this paper, sEMG data are decomposed into several IMF components, and empirical mode decomposition is stopped when the average frequency of IMF is less than $20 \mathrm{~Hz}$. Then, soft threshold is processed for each IMF, and its calculation formula is as follows: 


$$
\eta\left(\mathrm{IMF}_{\mathrm{ij}}\right)=\left\{\begin{array}{cc}
\left|\mathrm{IMF}_{\mathrm{ij}}\right|-\lambda_{i} & \left|\mathrm{IMF}_{\mathrm{ij}}\right| \geq \lambda_{i} \\
0 & \left|\mathrm{IMF}_{\mathrm{ij}}\right| \leq \lambda_{i}
\end{array}\right.
$$

where $\mathrm{IMF}_{\mathrm{ij}}$ is the $j$ th sample point in the $i$ th IMF component, and $\lambda_{i}$ is the threshold, which is set as twice the standard deviation of the first 1000 sample points of the signal.

Finally, the IMF decomposition after soft threshold is added linearly to obtain the reconstructed signal. Figure 3 is an example of the reconstructed signal. The noise interference of involuntary activity was effectively eliminated using the EMD algorithm.

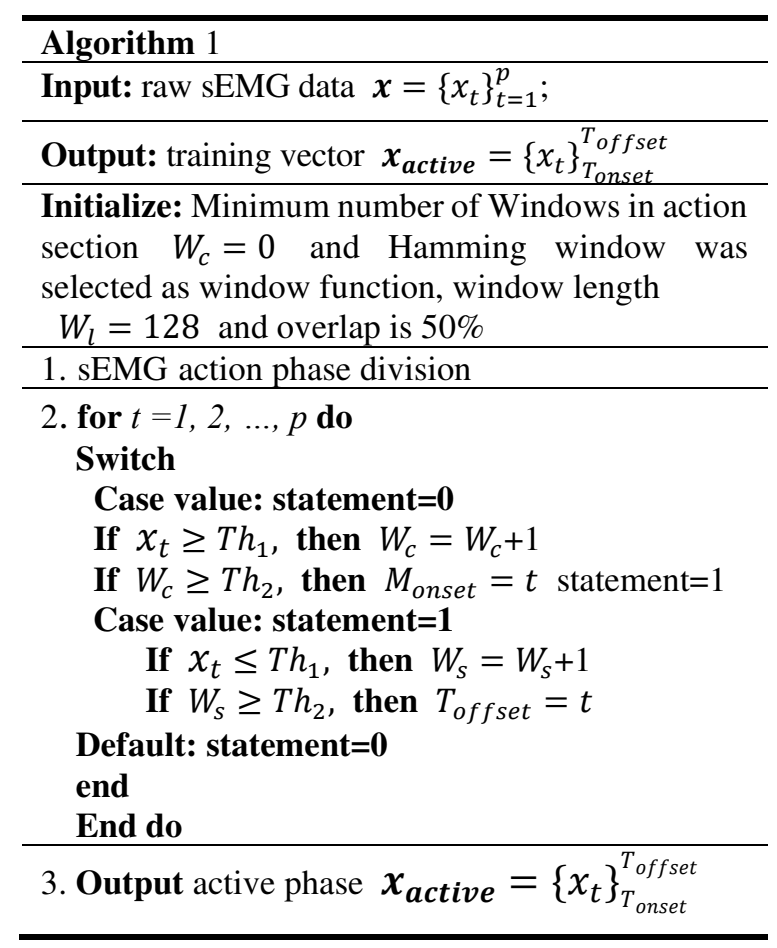

Action phase division and feature extraction

Different window lengths have been verified and recommended due to different classification problems and delay requirements [37]. Different window lengths $(32,64,128,256$ and $512 \mathrm{~ms})$ were investigated. The window length of this paper is 128 $\mathrm{ms}$. The degree of window overlap is independent of the classification error [37]. Therefore, in this paper, the window overlap is $50 \%$ as reported in [37] and [38].

The three muscles were segmented into active segments, and the motor process of each muscle was extracted. The muscle action phase division algorithm flow of sEMG is shown in Algorithm 1. When the signal exceeds the threshold value of $T h_{1}$, it is believed that the muscle may start to enter the active activation state. In this paper, $T h_{1}$ is set as the mean value of the whole signal after filtering.

$T h_{1}=\frac{1}{P} \sum_{t=0}^{P} x_{t}$
To ensure the accuracy of active activation and prevent the involuntary activation of the muscle from causing wrong division, double threshold values are adopted in this paper. The threshold $T h_{2}$ is used to eliminate the interference of noise to the action activation segment. The movement range of the experiment was approximately $80^{\circ}$. According to the experiment with healthy subjects, the slow movement speed of elbow joint of healthy subjects was concentrated at $60 \%$ s. Therefore, to prevent noise interference, the minimum duration of the activation section is set to be no less than 1 s, i.e., $T h_{2}=8$. After that, the IMU signal is smoothed with the sliding average algorithm. In addition, the activation segment signals of each muscle are extracted. During the IMU motion activation segment, sEMG data of each muscle were also extracted. The threshold method was used to judge the duration of active motion of IMU signals. The onset threshold is considered to enter active motion if it exceeds $10 \%$ of the maximum motion angle. The first derivative difference method is used to calculate the end time of active motion, i.e., the first derivative of three consecutive Windows is less than 0.01 . Figure 3 presents the signal diagram calculated by the action phase division algorithm. sEMG and IMU signals in the region of the action phase division algorithm were used for feature extraction and fusion.

For feature extraction of the motor activation segment, this paper extracted 4 common sEMG time domain (TD) features, 2 sEMG frequency domain (FD) features and 3 motion signal features. These features were the root mean square (RMS), variance (VAR), mean absolute value (MAV), waveform length (WL), median frequency (MNF), median frequency (MDF), movement angle (MA), angular velocity (AV) and angular acceleration (AA). RMS is the square root of the average power of the sEMG. MAV is the absolute value of the sEMG. VAR is a measure of the sEMG power. WL can reflect the complexity of the sEMG signal. MDF is an average frequency, which is calculated as the sum of products of the EMG power spectrum, and MDF is the frequency at which the spectrum is divided into two regions with equal amplitude [39]. The complementary filtering approaches [40] was used to calculate MA, AV is obtained by a gyroscope and the first-order difference equations of MA were calculated to obtain AA. The six sEMG features are described below:

$$
\begin{aligned}
& \operatorname{RMS}_{i}(t)=\sqrt{\frac{1}{M} \sum_{k=1}^{M} x_{i}^{t}(k)^{2}} \\
& \operatorname{VAR}_{i}(t)=\frac{1}{M-1} \sum_{k=1}^{M}\left(x_{i}^{t}(k)-\frac{1}{M} \sum_{k=1}^{M} x_{i}^{t}(k)\right)^{2} \\
& \operatorname{MAV}_{i}(t)=\frac{1}{M} \sum_{k=1}^{M}\left|x_{i}^{t}(k)\right| \\
& \left\{\begin{array}{l}
x_{i}^{t}(k)=\sum_{j=1}^{p} a_{j} x_{i}^{t}(k-j)+w_{i}^{t}(k) \\
\mathrm{WL}_{i}(t)=\sum_{k=1}^{M}\left|x_{i}^{t}(k+1)-x_{i}^{t}(k)\right|
\end{array}\right.
\end{aligned}
$$




$$
\begin{aligned}
& M N F_{i}(t)=\sum_{k=1}^{M} f_{j} P_{j} / \sum_{k=1}^{M} P_{j} \\
& M N F_{i}(t)=\sum_{k=1}^{M} P_{j} \\
& \mathrm{AV}(\mathrm{t})=\frac{1}{M} \sum_{k=1}^{M} \sqrt{I_{x}^{t}(k)^{2}+I_{y}^{t}(k)^{2}+I_{z}^{t}(k)^{2}} \\
& \left\{\begin{array}{l}
g y o(k)=I(k) / f s \\
M A(k)=\alpha * g y o(k)+(1-\alpha) \operatorname{acc}(k) \\
M A(t)=\frac{1}{M} \sum_{k=1}^{M} M A(k)
\end{array}\right.
\end{aligned}
$$

where $x_{i}^{t}(k)$ represents the $k$ th sEMG sample in the $t$ th overlapping analysis window in the $i$ th channel, $a_{j}$ is the $j$ th order autoregression (AR) coefficient, $p$ is the order of AR model $(p=4)$, $w_{i}^{t}(k)$ is the white noise residual, $f_{j}$ is frequency of the spectrum at frequency bin $\mathrm{j}$, and $P_{j}$ is the EMG power spectrum at frequency bin j. $I_{x}^{t}(k)^{2}, I_{y}^{t}(k)^{2}$ and $I_{z}^{t}(k)^{2}$ is the $k$ th gyroscope sample of $\mathrm{x}$ axis, $\mathrm{y}$ axis and $\mathrm{z}$ axis respectively. $\alpha$ is the filter coefficient, which set as 0.96 in this paper. $\operatorname{acc}(k)$ is the angle count by $k$ th accelerometer data.

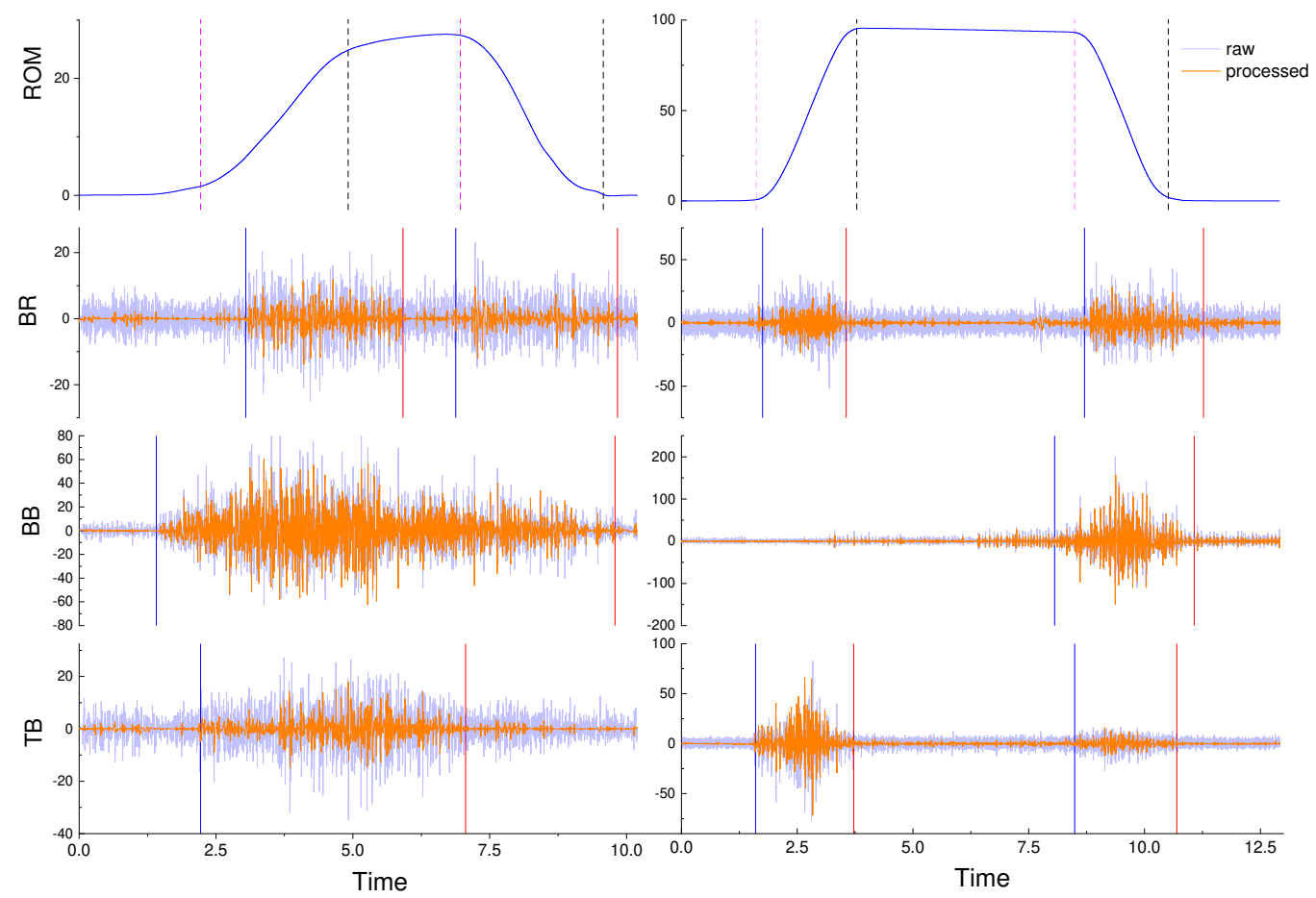

(a)

(b)

Fig. 3 Sample of a subject after the data processing algorithm. (a) ROM and three muscle sEMGs of stroke subject. (b) ROM and three muscle sEMGs of a healthy subject. The blue signal is the original sEMG, the orange signal is the signal after processing, and the blue curve is the motion angle. The pink dashed line is the starting point of the motion angle, and the black dash line indicates the end of the motion angle. The blue solid line represents the starting point of muscle activation, and red represents the end point of muscle activation.

\section{Classification scheme}

The k-nearest neighbor (k-NN) classifier [41], support vector machine (SVM)[42], random forest (RF) [43] and multilayer perceptron (MLP) were used to classify the spasticity of the participants.

The RF classifier analysis is a supervised method of the multiple decision trees. Given its good generalization and robustness to outliers and noise, it is widely used in the analysis of biological signals[44]. As shown in Figure 4, the main procedure of RF algorithm includes the following: (1) A random sample of the put back from the original data set is used to generate the subsets of which sizes are equal to that of the original set; (2) A subset of features was randomly selected from the reconstructed training set to construct decision trees; (3) Final results are obtained by fusing the prediction or classification results of all the decision trees. Thus, the output of each tree is considered a vote, with the majority vote determining the output of the random forest.

According to the characteristics of RF, the parameters to be optimized for this model include the number of RF neutron trees, the maximum depth of the decision tree, the minimum number of samples required for repartition of internal nodes, the minimum number of samples required for leaf nodes, and the maximum number of RF features allowed to be used by a single decision tree. In this paper, the gridsearchcv method is used to optimize the above parameters.

To test the performance of random forest, this paper selected another three common machine learning 
methods, including k-NN, SVM and MLP, for performance comparison. To make a fair comparison, parameter optimization is performed for the above three models. All models were modeled and optimized using PYTHON (version 3.6, Python Software Foundation, Hampton, NH).

\section{Performance evaluation of models}

To eliminate the influence of feature magnitude on the model, the feature is normalized. The following formula is used to normalize each feature:

$y_{\text {norm }}=\frac{x-x_{\min }}{x_{\max }-x_{\min }}$

Each dataset consisting of a recombined feature matrix and a label vector was still divided into a training set and test set at a ratio of 80:20. The 5-fold cross-validation method was performed to verify the performance of the different classifiers. We calculated the accuracy, recall and F1 score as the evaluation metrics. To use the F1 score for the evaluation of the multiclassification model, these metrics are calculated as follows[45],[46]:

$$
\left\{\begin{array}{l}
\text { Accuracy }=\frac{\mathrm{TP}+\mathrm{TN}}{\mathrm{TP}+\mathrm{TN}+\mathrm{FP}+\mathrm{FN}} \\
\text { Precision }=\frac{\mathrm{TP}}{\mathrm{TP}+\mathrm{FP}} \\
\text { Recall }=\frac{\mathrm{TP}}{\mathrm{TP}+\mathrm{FN}} \\
\mathrm{F} 1_{\text {macro }}=\frac{2 * \text { Precision } * \text { Recall }}{\text { Precision }+ \text { Recall }}
\end{array}\right.
$$

where TP, TN, FN, and FP are true positives, true Negative, false negatives, and false positives, respectively.

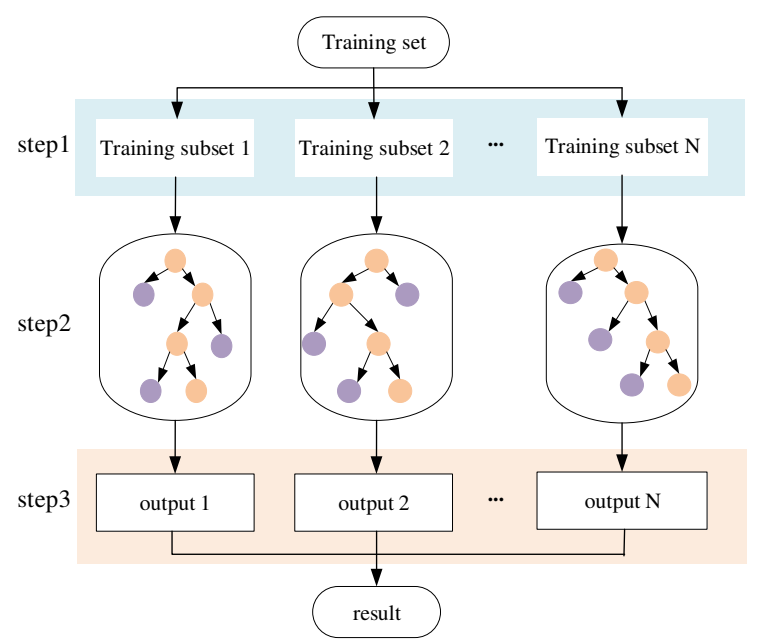

Fig. 4 Flow chart of random forest model training.

\section{Statistical analysis}

Statistical analysis was performed using IBM SPSS statistics software (ver. 24.0, IBM Corp., Armonk, NY, USA)). Differences in the F1-scores of the classification model were tested for statistical significance using a paired t-test. A significance level of $\mathrm{P}<0.05$ was used for all the analyses.

\section{Results \\ Model performance}

As shown in Table 2, the mean F1 score of all models was greater than 0.88 . The random forest model obtained the highest F1 score $(0.976 \pm 0.028)$, which was significantly higher than the other three models.

As shown in Table 3, the classification accuracy of each type of the four convulsion degrees of the four models is shown. RF obtained the highest classification accuracy in the four degrees of spasticity $(0.986,0.956,0.981$ and 0.972 respectively) among the four models. For the spasm degree of grade 0 (healthy), grade 1 , and grade $1+$, the classification accuracy of the four models was greater than 0.882 , and the classification accuracy of grade 0 spasm degree was greater than 0.95 . The minimum accuracy is $1+$ classification accuracy of $\mathrm{k}-\mathrm{NN}$ (0.882). However, for the classification of grade 2 spasticity, the classification accuracy of grade 2 spasticity in the three models was significantly reduced. The lowest accuracy is 0.735 in k-NN. Comparison of RF with the other three models is realized using a one-way analysis of variance (ANOVA) followed by the post hoc Tukey test with a significance level of 0.05 . there is statistically significant difference among RF to the other three models in four spasticity grades $(\mathrm{p}<0.001)$.

Table 2 Average classification performance of Classification models

\begin{tabular}{cccc}
\hline Model & Accuracy & Recall & F1-score \\
\hline k-NN & 0.880 & 0.880 & 0.880 \\
SVM & 0.911 & 0.890 & 0.896 \\
RF & 0.979 & 0.974 & 0.976 \\
MLP & 0.930 & 0.931 & 0.929 \\
\hline
\end{tabular}

Table 3 Average accuracy performance of spasticity degree in Classification models

\begin{tabular}{ccccc}
\hline model & 0 & 1 & $1+$ & 2 \\
\hline k-NN & 0.926 & 0.923 & 0.882 & 0.735 \\
SVM & 0.950 & 0.893 & 0.939 & 0.780 \\
RF & 0.986 & 0.956 & 0.981 & 0.972 \\
MLP & 0.967 & 0.930 & 0.939 & 0.887
\end{tabular}

To show the classification error in more detail, the confusion matrix of the four models is shown in Figure 5. The confusion matrix of the models shows that the max misclassification of grade 2 is grade $1+$. 
The k-NN model has 0.147 grade 2 that is misclassified into grade $1+, 0.177$ in RF, and 0.085 in MLP. Although there is some confusion between grade $1+$ and grade 2 , the RF average sorting accuracy is 0.956 , demonstrating a good ability to distinguish grade $1+$ from grade 2 .

\section{Feature importance}

We analyzed the importance of muscles and features, separately. The importance of the muscle is the sum of the importance ratios of the 9 features of the muscle. The importance of the trait is the sum of the proportions of the three muscles in the trait. As shown in Figure 6 (a), during flexion and extension, the importance of brachioradialis muscle and brachioradialis triceps varies greatly, while the biceps muscle does not. During flexion, the ratio of

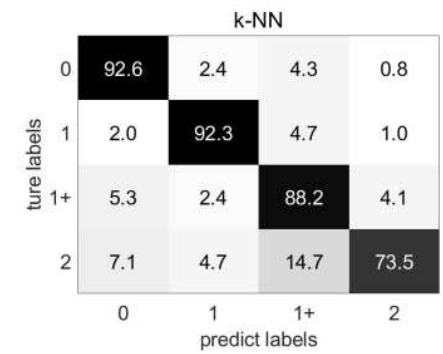

(a)

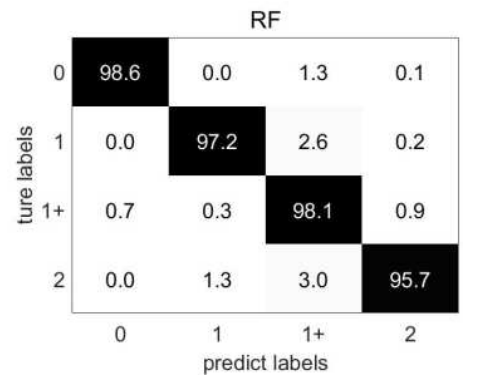

(c) importance of brachioradialis, biceps and triceps was $0.421,0.361$ and 0.218 , respectively. During the elbow stretching process, the values were $0.264,0.370$ and 0.367 , respectively. Therefore, in the process of elbow flexion and extension, the active muscle feature is the main factor muscle for evaluating spasm degree. However, whether the flexor extensor spasm is evaluated, the biceps feature is essential. As the shown in Figure 6 (b), the importance of features is not affected by the process of elbow motion; thus, the importance of features is not affected by the process of flexion and extension. The 4 TD sEMG features (0.46) dominate the feature importance. The proportion of kinematic features is 0.38 , and FD features accounted for the lowest proportion at 0.16 . The results show that FD features minimally influence the evaluation of spasmodic grade.

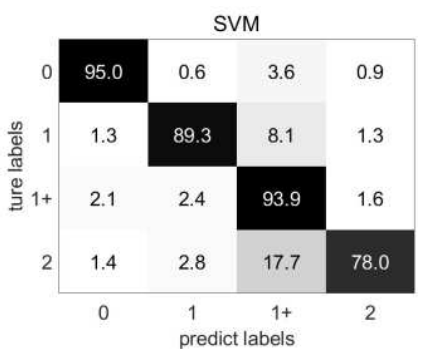

(b)

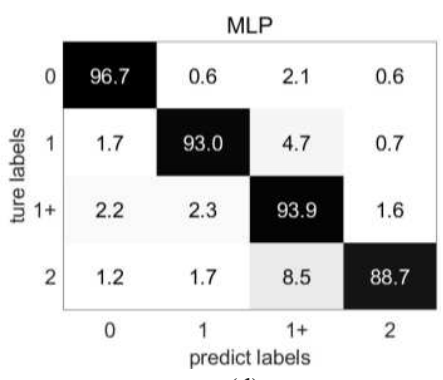

(d)

Figure 5 Confusion matrix of Classification models. a) k-NN classifier with an accuracy of 0.880. b) SVM classifier with an accuracy of 0.896. c) MLP classifier with an accuracy of 0.929. d) RF classifier with an accuracy of 0.976 . The confusion matrix element is expressed as a percentage, and the sum of the elements of each row is equal to 100. The diagonal of the matrix is the correct classification.

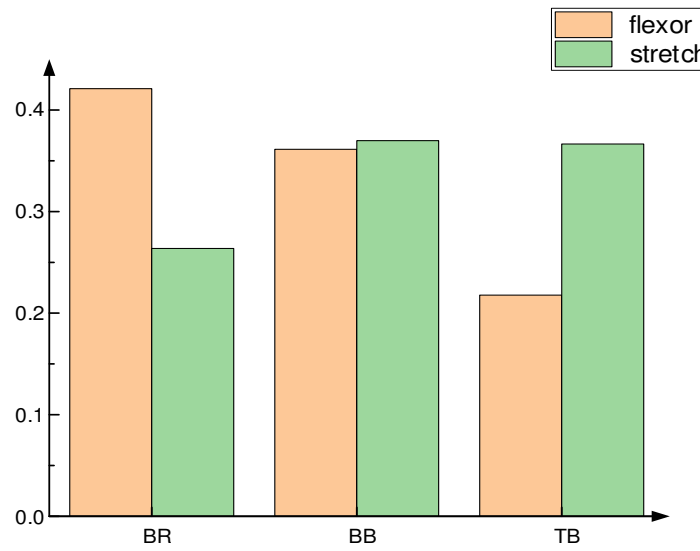

(a) muscle importance

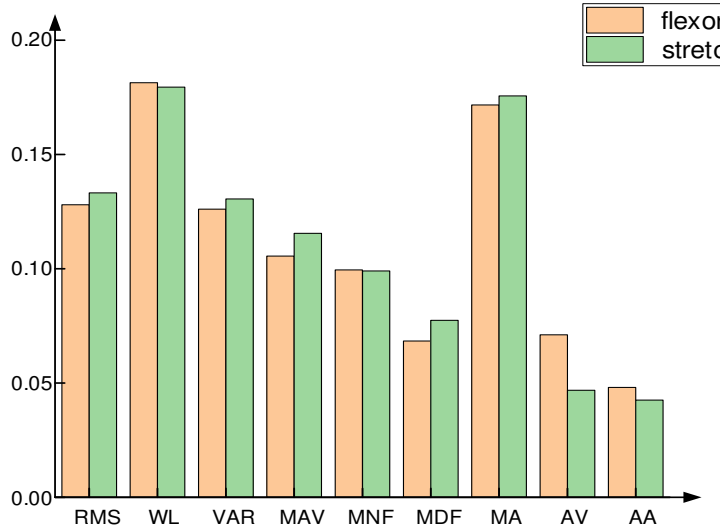

(b) feature importance 
Figure 6 Variable importance of random forest model. (a) Importance of muscle features (BR: brachioradialis muscle, BB: biceps brachialis; TB: triceps brachialis). (b) Importance of the feature. The IMU is the feature importance sum of MA, AV and AA.

\section{Discussion}

In this paper, we introduced a novel method to evaluate spasmodic state under voluntary motion. First, the EMD algorithm is used to filter the sEMG data to remove the involuntary sEMG caused by spasticity. Then, a double threshold algorithm is used to extract the active motion segment. Feature extraction was performed on the active motion segment, and fusion analysis was performed on the motion feature and surface sEMG feature. Finally, four commonly used models are used to classify and verify the features extracted in this paper, and the results show that the four models can effectively classify the spasm degree into the four types.

As shown in Figure 6, TD feature of sEMG dominate the feature importance. During elbow active action, different contributions of brachioradialis and triceps brachii indicate that the co-activation mode of active and antagonistic muscles is the main factor to evaluate the degree of spasticity under active motion. results show that co-activation mode is not only the main feature of spasm recognition in passive motion [32], but also dominates in active motion. That is to say, the degree of spasm under active motion is correlated with that of passive motion [29]. As shown in Fig. 3, healthy subjects can rest at the maximum elbow position, while the patient's signal is more disordered. Although the patient feels the muscles relax, they show greater activation. The reason for this is that at the maximum elbow position, active muscles need to generate the force that works against spastic muscles, resulting in more intense muscle activation. When the patient's active movement exceeds the threshold of the stretch reflex he/she can regulate [28], he/she shows abnormal muscle activation and thus produces an abnormal co-activation pattern. Therefore, our method can effectively capture the onset and offset time of abnormal muscle activation under active motion, which is the reason for the high classification accuracy of the model in this paper.

As shown in Figure 5, all models show lower classification accuracy of spasticity grade 2 than other spasticity grades, which are easily misclassified into grade $1+$. The reason may be that the patient was accompanied by grade 2 spasm and had compensatory exercise during the experiment. Abnormal coordination is one of the factors that cause dyskinesia of reaching movement [28]. In the process of maximum flexion and extension of the elbow, to achieve the maximum movement state as expected by himself, the patient uses trunk compensation for elbow joint displacement to generate a larger motion angle. The difference between its kinematics characteristics and the kinematics feature of 1+ grade spasticity is reduced, which leads to misclassification.
Although the incomplete limitation of the elbow joint caused the classification error, both RF and MLP have good resolution accuracy in spasticity grade 2 . Given that the classification of MLP reflected the black box effect, it was difficult to analyze the importance of features, making it difficult for researchers to further analyze the pathological characteristics of spasticity and extract more applicable clinical evaluation factors based on the current model. RFs allow us to examine the training procedure and quantify the importance of different features to the classification task [47]. Therefore, we recommended using the Random Forest algorithm to model the classification of spasticity degree. The classification of the model is helpful to the retrograde pathological mechanism.

The pathological mechanism of spasm remains to be studied. [12] suggested that spasticity should be replaced by hyper-resistance and that the spastic nerve parts not only exhibit hyperreflexia but also retain involuntary muscle activation. Involuntary muscle activation enhanced the background noise. [48] notes that normal people in active exercise will adjust the threshold of stretch reflex to inhibit hyper-reflexes in the active process. The spasmodic threshold, which is measured at rest in a spasmodic patient, is adjusted during active motion. Isolation of involuntary muscle activation during voluntary movement is helpful to study the abnormal muscle activity caused by stretch reflex during voluntary movement. Therefore, the study of spasticity under active motion is helpful to the study of the pathological mechanism of spasm. In this paper, the importance of frequency-domain features in the classification of spasm is low. Feature importance still remains at 0.84 if the FD feature is removed. This phenomenon may indicate that the involuntary muscle activation of spasticity is less related to the firing rate of muscle fibers in the process of active exercise and more related to the recruitment mode of motor units. Meanwhile, low importance of TD feature can reduce the computing required for wearables. If the computing performance of embedded devices is limited, the FD feature can be removed.

Overall, this study had a number of strengths. First, the fusion of sEMG and kinematic signals verifies the feasibility of evaluating spasm under active motion. Second, the machine learning algorithm is used to detect and classify the degree of spasm. Finally, the influence of random forest features on the classification of spasm is analyzed based on the importance of random forest features. Results demonstrate that our method could be potentially applied to spasticity assessment during rehabilitation exercise training. 


\section{Future Work}

Wearable assessment devices can assist in assessing patients' spasmodic states during rehabilitation training, thus reminding patients to adjust their movements and reducing therapist workload. In future work, we will study the real time algorithm for spasticity assessment and apply it to wearable devices. Moreover, the motion of the upper limbs of the human body is mainly a complex motion in 3 dimensions. The plane movement of the fixed track mainly occurs in rehabilitation training. As the patient's condition eases, the patient will be trained in daily life movements. In future work, we will study the methods of assessing upper limb spasm in patients with daily life movements to make the wearable assessment devices suitable for family scenes.

\section{Conclusion}

In summary, we propose a novel method for the separation of voluntary components and involuntary activation components of electromyographic signals in patients with spasticity under active motion and verified the feasibility of evaluating spasticity under active exercise. The RF algorithms exhibited excellent classification performance in detecting and categorizing four grades of spasticity for all nine participants in this study (F1-score=0.976). The assessment of spasticity based on active exercise provides the possibility for the assessment of rehabilitation training for stroke survivors and home training. The assessment of spasticity based on active exercise provides the possibility for the assessment of rehabilitation training for stroke survivors and home training, which can help therapists adjust rehabilitation strategies and achieve more targeted and personalized rehabilitation programs. Future work will focus on developing an effective wearable spasmodic assessment system for stroke survivors to assess spasmodic states in rehabilitation training.

\footnotetext{
Abbreviations

MAS: Modified Ashworth Scale; ROM: range of motion; IMU: inertial measurement unit; sEMG: surface electromyography; MMSE: Mini Mental State Examination; VAS: Visual Analogue Scale; BR: brachioradialis muscle; BB: biceps brachialis; TB: triceps brachialis; EMD: empirical mode decomposition; TD: time domain; FD: frequency domain; RMS: root mean square; VAR: variance; MAV: mean absolute value; WL: waveform length; MNF: median frequency; MDF: median frequency, MA: movement angle; AV: angular velocity; AA: angular acceleration; k-NN: k-nearest neighbor; SVM: support vector machine; RF: random forest; MLP: multilayer perceptron.
}

\section{Acknowledgements}

The authors would like to thank all participants, the occupational therapists and all the doctors in the rehabilitation department of The Third Affiliated Hospital of Sun Yat-sen University.

\section{Availability of data and materials}

The datasets used and/or analyzed during the current study are available from the corresponding author upon reasonable request.

\section{Authors' contributions}

YC developed the data processing algorithms, trained and evaluated the machine learning classifiers, and drafted the manuscript. SY participated in the experimental design, data collection and data preprocess. QC recruited subjects and participated in the data collection. SH participated experimental data collection and revised the manuscript. KM participated experimental data collection. HZ participated in the study design. LX participated in the study design and revised the manuscript. All the authors read and approved the final manuscript.

\section{Funding}

National Natural Science Foundation of China (grant no. 51575188), National Key R\&D Program of China (grant no. 2018YFB1306201), Research Foundation of Guangdong Province (grant nos. 2016A030313492 and 2019A050505001), and Guangzhou Research Foundation (grant no. 201903010028). The funders had no role in study design, data collection and analysis, decision to publish, or preparation of the manuscript.

\section{Ethics approval and consent to participate} Ethics approval and consent to participate (i.e., informed consent) was obtained from all the participants to complete the protocol approved by the Guangzhou First People's Hospital Department of Ethics Committee. All the research was performed in accordance with the Declaration of Helsinki.

\section{Consent for publication}

Not applicable.

\section{Competing interests}

The authors declare that they have no competing interests.

\section{References}

1. Dressler D, Bhidayasiri R, Bohlega S, Chana P, Chien HF, Chung TM, et al. Defining spasticity: a new approach considering current movement disorders terminology and botulinum toxin therapy. $\mathbf{J}$ Neurol. 2018;265:856-62. 
2. Pandyan A, Gregoric M, Barnes M, Wood D, Wijck FV, Burridge J, et al. Spasticity: Clinical perceptions, neurological realities and meaningful measurement. Disability and Rehabilitation. 2005;27:2-6.

3. Brunnstrom S. Motor Testing Procedures in Hemiplegia: Based on Sequential Recovery Stages. Physical Therapy. 1966;46:357-75.

4. Naghdi S, Ansari NN, Mansouri K, Hasson S. A neurophysiological and clinical study of Brunnstrom recovery stages in the upper limb following stroke. Brain Injury. 2010;24:1372-8.

5. Brueckner D. Associations Between PracticeRelated Changes in Motor Performance and Muscle Activity in Healthy Individuals: A Systematic Review. 2018;10.

6. Li S. Spasticity, Motor Recovery, and Neural Plasticity after Stroke. Front Neurol [Internet]. 2017 [cited $2020 \mathrm{Feb} 10] ; 8$. Available from: http://journal.frontiersin.org/article/10.3389/fneur.20 17.00120/full

7. Gregson JM, Leathley M, Moore AP, Sharma AK, Smith TL, Watkins CL. Reliability of the tone assessment scale and the modified ashworth scale as clinical tools for assessing poststroke spasticity. Archives of Physical Medicine and Rehabilitation. 1999;80:1013-6.

8. Pandyan AD, Johnson GR, Price CIM, Curless RH, Barnes MP, Rodgers H. A review of the properties and limitations of the Ashworth and modified Ashworth Scales as measures of spasticity. Clin Rehabil. 1999;13:373-83.

9. Fleuren JFM, Voerman GE, Erren-Wolters CV, Snoek GJ, Rietman JS, Hermens HJ, et al. Stop using the Ashworth Scale for the assessment of spasticity. Journal of Neurology, Neurosurgery \& Psychiatry. 2010;81:46-52.

10. Thibaut A, Chatelle C, Ziegler E, Bruno M-A, Laureys S, Gosseries O. Spasticity after stroke: Physiology, assessment and treatment. Brain Injury. 2013;27:1093-105.

11. van den Noort JC, Bar-On L, Aertbeliën E, Bonikowski M, Braendvik SM, Broström EW, et al. European consensus on the concepts and measurement of the pathophysiological neuromuscular responses to passive muscle stretch. Eur J Neurol. 2017;24:981-e38.

12. Eby S, Zhao H, Song P, Vareberg BJ, Kinnick R, Greenleaf JF, et al. Quantitative Evaluation of Passive Muscle Stiffness in Chronic Stroke: American Journal of Physical Medicine \& Rehabilitation. 2016;95:899-910.

13. Hugos CL, Cameron MH. Assessment and Measurement of Spasticity in MS: State of the Evidence. Curr Neurol Neurosci Rep. 2019;19:79.
14. Ang WS, Geyer H, Chen I-M, Ang WT. Objective Assessment of Spasticity With a Method Based on a Human Upper Limb Model. IEEE Trans Neural Syst Rehabil Eng. 2018;26:1414-23.

15. Choi S, Shin YB, Kim S-Y, Kim J. A novel sensor-based assessment of lower limb spasticity in children with cerebral palsy. J NeuroEngineering Rehabil. 2018;15:45.

16. Centen A, Lowrey CR, Scott SH, Yeh T-T, Mochizuki G. KAPS (kinematic assessment of passive stretch): a tool to assess elbow flexor and extensor spasticity after stroke using a robotic exoskeleton. J NeuroEngineering Rehabil. 2017;14:59.

17. Çakır T, Evcik FD, Subaşı V, Demirdal ÜS, Kavuncu V. Investigation of the $\mathrm{H}$ reflexes, $\mathrm{F}$ waves and sympathetic skin response with electromyography (EMG) in patients with stroke and the determination of the relationship with functional capacity. Acta Neurol Belg. 2015;115:295-301.

18. Eby S, Zhao H, Song P, Vareberg BJ, Kinnick R, Greenleaf JF, et al. Quantitative Evaluation of Passive Muscle Stiffness in Chronic Stroke: American Journal of Physical Medicine \& Rehabilitation. 2016;95:899-910.

19. Leng Y, Wang Z, Bian R, Lo WLA, Xie X, Wang R, et al. Alterations of Elastic Property of Spastic Muscle With Its Joint Resistance Evaluated From Shear Wave Elastography and Biomechanical Model. Front Neurol. 2019;10:736.

20. Zhang X, Tang X, Zhu X, Gao X, Chen X, Chen $X$. A Regression-Based Framework for Quantitative Assessment of Muscle Spasticity Using Combined EMG and Inertial Data From Wearable Sensors. Front Neurosci. 2019;13:398.

21. Hu B, Zhang X, Mu J, Wu M, Zhu Z, Liu Z, et al. Spasticity Measurement Based on the HHT Marginal Spectrum Entropy of sEMG Using a Portable System: A Preliminary Study. IEEE Trans Neural Syst Rehabil Eng. 2018;26:1424-34.

22. Turpin NA, Feldman AG, Levin MF. Stretchreflex threshold modulation during active elbow movements in post-stroke survivors with spasticity. Clinical Neurophysiology. 2017;128:1891-7.

23. Lindberg PG, Gäverth J, Islam M, Fagergren A, Borg J, Forssberg H. Validation of a New Biomechanical Model to Measure Muscle Tone in Spastic Muscles. Neurorehabil Neural Repair. 2011;25:617-25.

24. Li Y, Zhang X, Gong Y, Cheng Y, Gao X, Chen $X$. Motor Function Evaluation of Hemiplegic UpperExtremities Using Data Fusion from Wearable Inertial and Surface EMG Sensors. Sensors. 2017; 17:582. 
25. Akpinar P, Atici A, Ozkan FU, Aktas I, Kulcu DG, Sar1 A, et al. Reliability of the Modified Ashworth Scale and Modified Tardieu Scale in patients with spinal cord injuries. Spinal Cord. 2017;55:944-9.

26. Misgeld BJE, Luken M, Heitzmann D, Wolf SI, Leonhardt S. Body-Sensor-Network-Based Spasticity Detection. IEEE J Biomed Health Inform. 2016;20:748-55.

27. Lance JW. Symposium synopsis. Spasticity: disordered motor control. 1980;487-489.

28. McPherson JG, Stienen AH, Drogos JM, Dewald JP. Modification of Spastic Stretch Reflexes at the Elbow by Flexion Synergy Expression in Individuals With Chronic Hemiparetic Stroke. Archives of Physical Medicine and Rehabilitation. 2018;99:491500.

29. Ellis MD, Schut I, Dewald JPA. Flexion synergy overshadows flexor spasticity during reaching in chronic moderate to severe hemiparetic stroke. Clinical Neurophysiology. 2017;128:1308-14.

30. Wang H, Huang P, Li X, Samuel OW, Xiang Y, Li G. Spasticity Assessment Based on the Maximum Isometrics Voluntary Contraction of Upper Limb Muscles in Post-stroke Hemiplegia. Front Neurol. 2019;10:465.

31. Mochizuki G, Centen A, Resnick M, Lowrey C, Dukelow SP, Scott SH. Movement kinematics and proprioception in post-stroke spasticity: assessment using the Kinarm robotic exoskeleton. Journal of neuroengineering and rehabilitation. 2019;16:146.

32. Kersten P, Küçükdeveci AA, Tennant A. The use of the Visual Analogue Scale (VAS) in rehabilitation outcomes. Journal of rehabilitation medicine. 2012;44:609.

33. Charalambous CP. Interrater reliability of a modified Ashworth scale of muscle spasticity. Classic papers in orthopaedics. Springer; 2014. p. 415-417.

34. Hermens HJ, Freriks B, Merletti R, Stegeman D, Blok J, Rau G, et al. European recommendations for surface electromyography. Roessingh research and development. 1999;8:13-54.

35. Konrad P. The abc of emg. A practical introduction to kinesiological electromyography. 2005; 1:30-35.

36. Silva MB, Silva AN, Naves ELM, Palomari ET, Soares AB. An improved approach for measuring the tonic stretch reflex response of spastic muscles. Computers in Biology and Medicine. 2017;80:16674.

37. Menon R, Di Caterina G, Lakany H,

Petropoulakis L, Conway BA, Soraghan JJ. Study on interaction between temporal and spatial information in classification of EMG signals for myoelectric prostheses. IEEE Transactions on Neural Systems and Rehabilitation Engineering. IEEE;

2017;25:1832-1842.

38. Chu J-U, Moon I, Mun M-S. A real-time EMG pattern recognition system based on linear-nonlinear feature projection for a multifunction myoelectric hand. IEEE Transactions on biomedical engineering. IEEE; 2006;53:2232-2239.

39. Phinyomark A, Phukpattaranont P, Limsakul C. Feature reduction and selection for EMG signal classification. Expert Systems with Applications. 2012;39:7420-31.

40. Tayebi A, McGilvray S, Roberts A, Moallem M. Attitude estimation and stabilization of a rigid body using low-cost sensors. 2007 46th IEEE Conference on Decision and Control. IEEE; 2007. p. 6424-6429.

41. Celisse A, Mary-Huard T. Theoretical analysis of cross-validation for estimating the risk of the knearest neighbor classifier. The Journal of Machine Learning Research. JMLR. org; 2018;19:2373-2426. 42. Ma J, Krishnamurthy A, Ahalt S. SVM training with duplicated samples and its application in SVMbased ensemble methods. Neurocomputing. Elsevier; 2004;61:455-459.

43. Chen W, Li Y, Xue W, Shahabi H, Li S, Hong H, et al. Modeling flood susceptibility using data-driven approaches of naïve Bayes tree, alternating decision tree, and random forest methods. Science of The Total Environment. 2020;701:134979.

44. Dwivedi A, Kwon Y, McDaid AJ, Liarokapis M. A Learning Scheme for EMG Based Decoding of Dexterous, In-Hand Manipulation Motions. IEEE Trans Neural Syst Rehabil Eng. 2019;27:2205-15. 45. Spiewak C, Islam M, Zaman MA-U. A Comprehensive Study on EMG Feature Extraction and Classifiers. Open Access J. Biomed Eng Biosci. 2018;1:17-26.

46. AbdelMaseeh M, Chen T-W, Stashuk DW. Extraction and classification of multichannel electromyographic activation trajectories for hand movement recognition. IEEE Transactions on Neural Systems and Rehabilitation Engineering. IEEE; 2015;24:662-673.

47. Mahapatra D. Analyzing Training Information From Random Forests for Improved Image Segmentation. IEEE Trans on Image Process. 2014;23:1504-12.

48. Levin MF. Principles of Motor Recovery After Neurological Injury Based on a Motor Control Theory. In: Laczko J, Latash ML, editors. Progress in Motor Control [Internet]. Cham: Springer International Publishing; 2016 [cited 2020 Mar 22]. p. 121-40. Available from: http://link.springer.com/10.1007/978-3-319-473130_7 
Figures

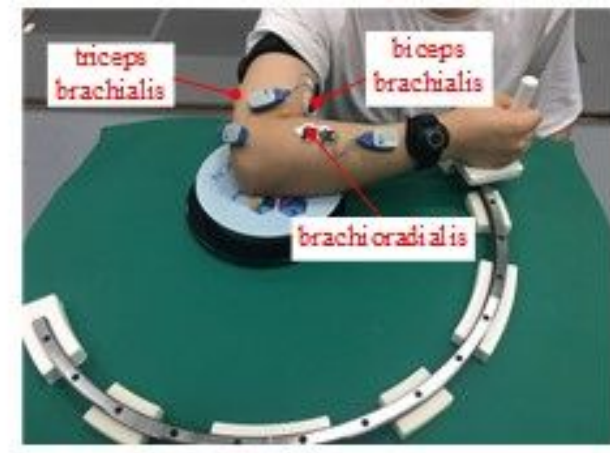

(a)

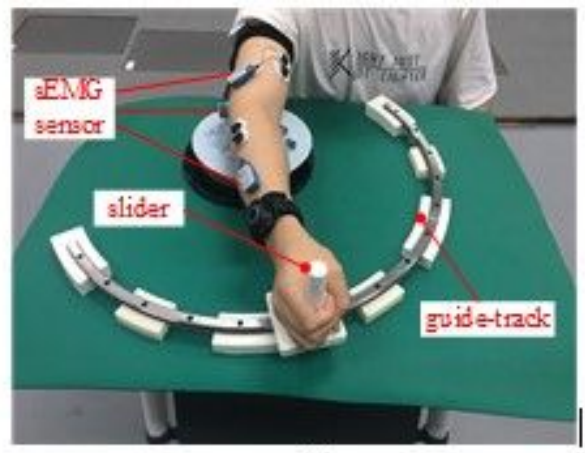

(b)

\section{Figure 1}

Flowchart of the proposed methodology. (a) The position of the surface electrodes placed on the upper limb. (b) The experimental setup.

Data Preprocessing

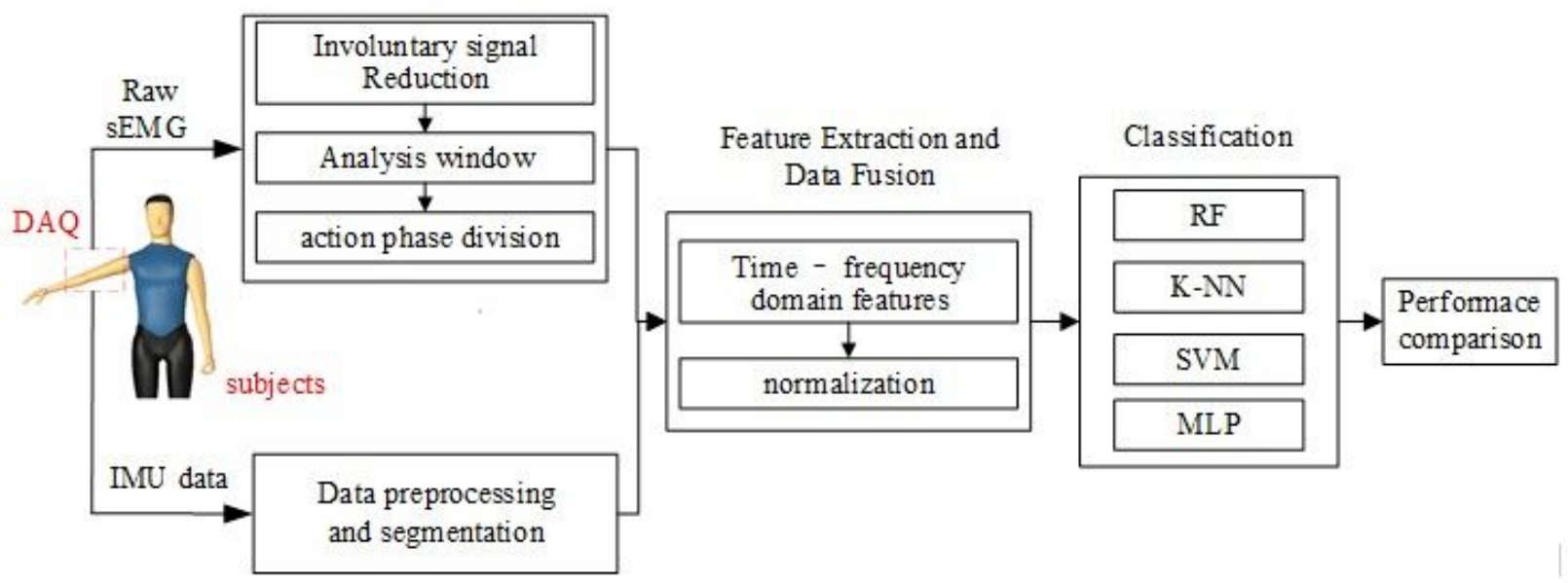

\section{Figure 2}

Flowchart of the proposed methodology. 


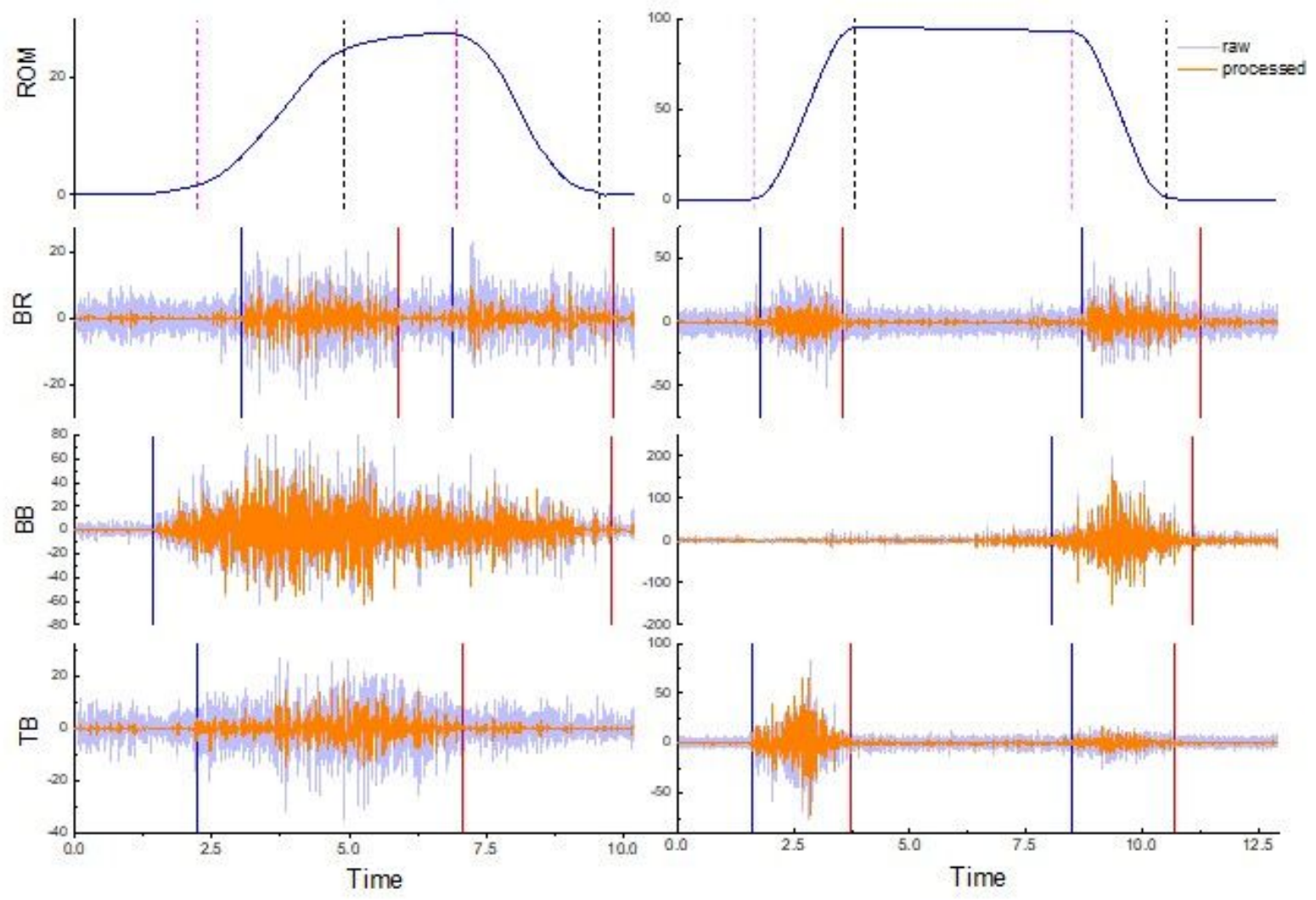

(a)

(b)

\section{Figure 3}

Sample of a subject after the data processing algorithm. (a) ROM and three muscle sEMGs of stroke subject. (b) ROM and three muscle sEMGs of a healthy subject. The blue signal is the original sEMG, the orange signal is the signal after processing, and the blue curve is the motion angle. The pink dashed line is the starting point of the motion angle, and the black dash line indicates the end of the motion angle. The blue solid line represents the starting point of muscle activation, and red represents the end point of muscle activation.

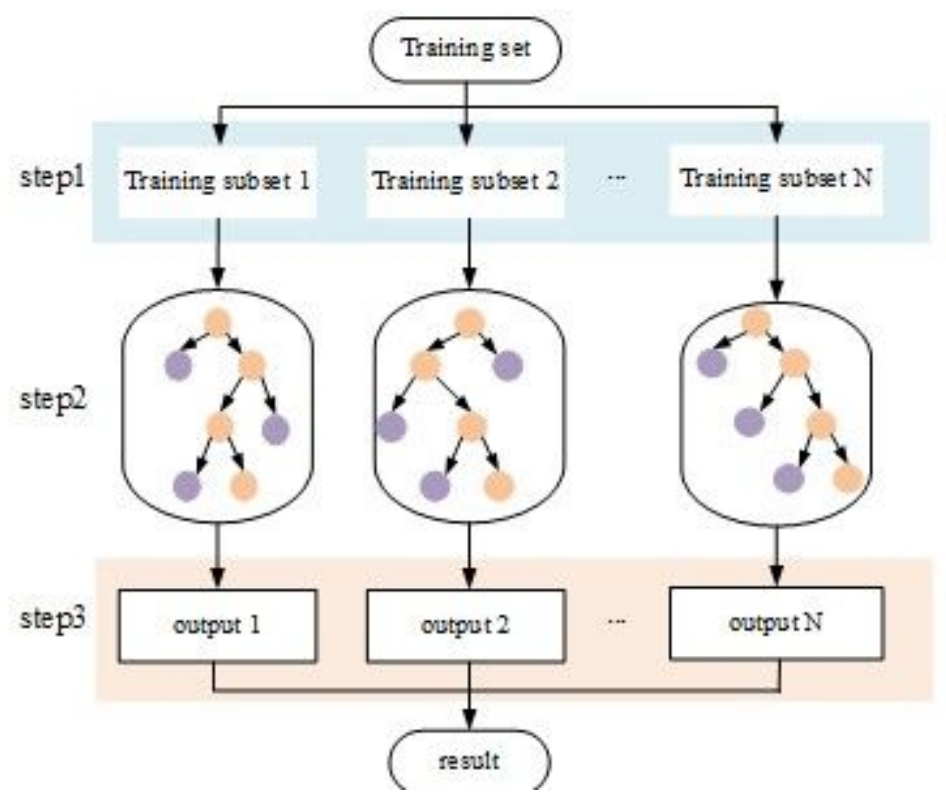


Figure 4

Flow chart of random forest model training

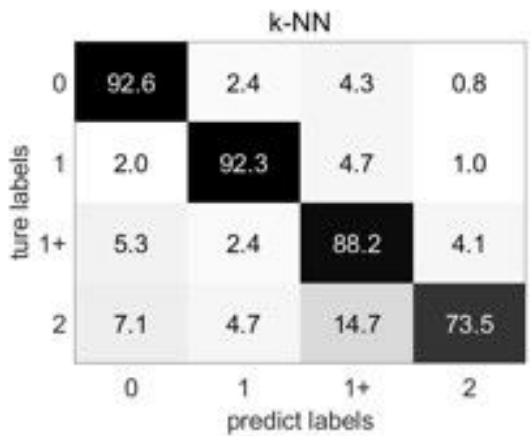

(a)

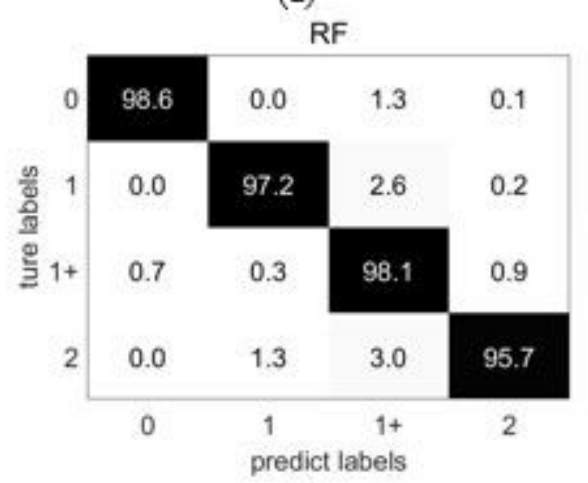

(c)

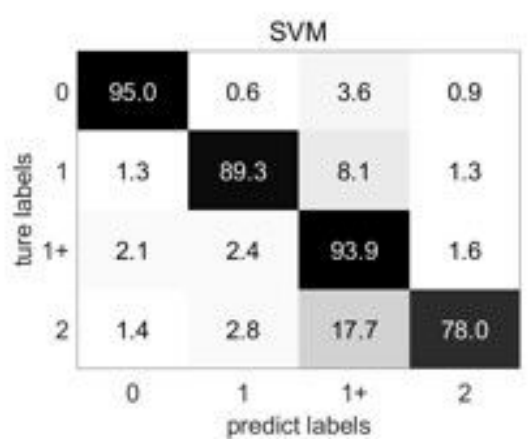

(b)

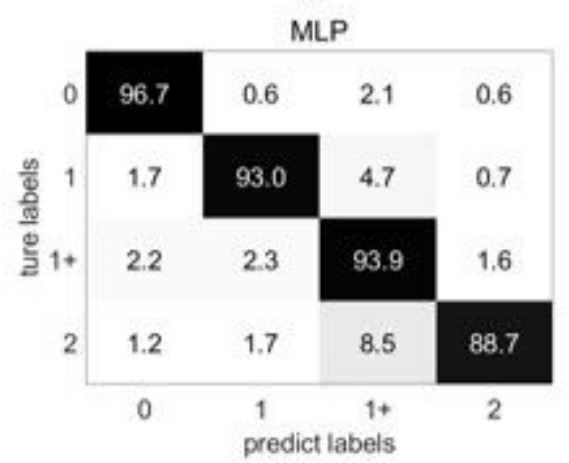

(d)

\section{Figure 5}

Confusion matrix of Classification models. a) k-NN classifier with an accuracy of 0.880 . b) SVM classifier with an accuracy of 0.896. c) MLP classifier with an accuracy of 0.929. d) RF classifier with an accuracy of 0.976 . The confusion matrix element is expressed as a percentage, and the sum of the elements of each row is equal to 100 . The diagonal of the matrix is the correct classification. 


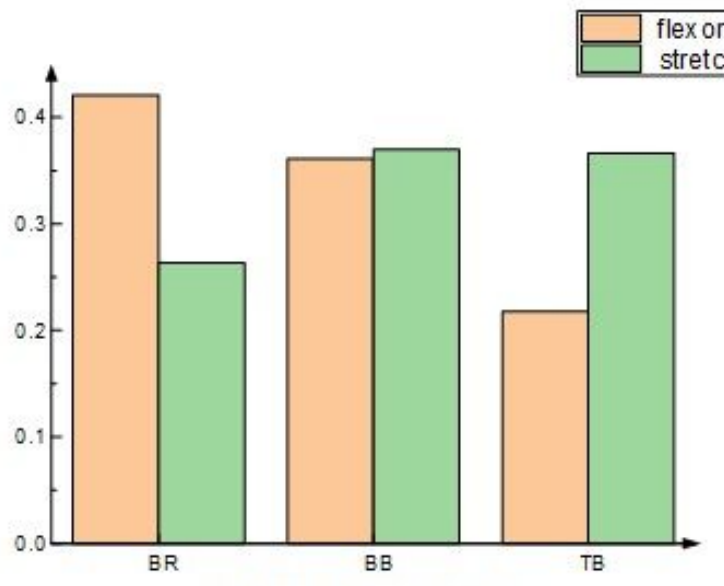

(a) muscle importance

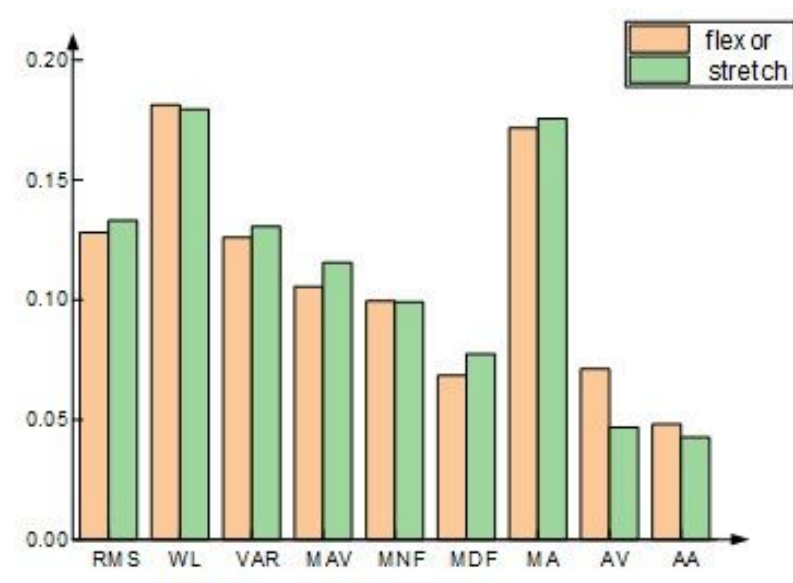

(b) feature importance

\section{Figure 6}

Variable importance of random forest model. (a) Importance of muscle features (BR: brachioradialis muscle, BB: biceps brachialis; TB: triceps brachialis). (b) Importance of the feature. The IMU is the feature importance sum of MA, AV and AA. 